\title{
ON THE USE OF EQUISPACED DISCRETE DISTRIBUTIONS
}

\author{
BY \\ J.F. WALHIN*• AND J. PARIS* \\ * Institut de Statistique, Université Catholique de Louvain, Belgium \\ - Le Mans Assurances, Belgique
}

\begin{abstract}
The Kolmogorov distance is used to transform arithmetic severities into equispaced arithmetic severities in order to reduce the number of calculations when using algorithms like Panjer's formulae for compound distributions. An upper bound is given for the Kolmogorov distance between the true compound distribution and the transformed one. Advantages of the Kolmogorov distance and disadvantages of the total variation distance are discussed. When the bounds are too big, a BerryEsseen result can be used. Then almost every case can be handled by the techniques described in this paper. Numerical examples show the interest of the methods.
\end{abstract}

\section{KEYWORDS}

Recursive formula; compound distribution; infinite divisibility; equispaced discrete distribution; Kolmogorov distance; total variation distance; upper and lower bounds; Berry-Esseen bound.

\section{INTRODUCTION}

The total claims distribution of a risk is given by

$$
S=X_{1}+\ldots+X_{N}
$$

where $\mathrm{X}$ denotes the severity of the claim distribution and $\mathrm{N}$ the claim frequency distribution. We suppose that the claim severity has a discrete probability function $f_{X}(x)$ and the claim frequency distribution has a probability function $p(n)$. 
The evaluation of the probability distribution $S$ is difficult since it needs the evaluation of convolutions of order $n$ and an infinite summation:

$$
f_{S}(x)=\sum_{n=0}^{\infty} p(n) f_{X}^{*^{\prime \prime}}(x)
$$

De Pril (1985) has given a recursive algorithm for the evaluation of the convolutions of arithmetic severities:

$$
\begin{gathered}
f_{X}^{*^{n}}(0)=f_{X}^{n}(0) \\
f_{X}^{*^{n}}(x)=\frac{1}{f_{X}(0)} \sum_{i=1}^{x}\left(\frac{n+1}{x} i-1\right) f_{X}(i) f_{X}^{*^{n}}(x-i) \quad x \geq 1
\end{gathered}
$$

Under the hypothesis that the claim frequency distribution satisfies the recursion:

$$
p(n)=p(n-1)\left(a+\frac{b}{n}\right) \quad n \geq 1
$$

Panjer (1981) has given a recursive algorithm for the evaluation of the compound distribution:

$$
\begin{gathered}
f_{S}(0)=\Psi_{N}\left(\ln f_{X}(0)\right) \\
f_{S}(x)=\frac{1}{1-a f_{X}(0)} \sum_{i=1}^{x}\left(a+b \frac{i}{x}\right) f_{X}(i) f_{S}(x-i) \quad x \geq 1
\end{gathered}
$$

where $\Psi_{N}(z)=\mathbb{E} e^{z N}$ denotes the moment generating function of $N$.

For both the recursions (4) and (7), the computing time will be significantly reduced if the claim severity is equispaced because in this case working on $0, h, 2 h, \ldots$ is the same as working on $0,1,2, \ldots$

Our goal is to replace the evaluation of

$$
\mathbb{P}[S \leq a]
$$

by the evaluation of

$$
\mathbb{P}\left[S_{a p p} \leq a\right]
$$

and to measure the difference between the two by the Kolmogorov distance.

The concepts discussed hereafter will be illustrated with the Poisson distribution. Of course the other laws satisfying (5) may be used as well as more complicated distributions satisfying more complicated recursions (see section 8). 
The following hypothetical claim severity distribution will be used for illustration:

TABLE 1

Claim SEVERITY DISTRIBUTION

\begin{tabular}{llllllllllll}
\hline \hline$X$ & 0 & 7 & 12 & 17 & 21 & 23 & 28 & 39 & 46 & 53 & 67 \\
$f_{X}$ & 0.05 & 0.1 & 0.15 & 0.05 & 0.05 & 0.05 & 0.1 & 0.1 & 0.1 & 0.15 & 0.1 \\
\hline
\end{tabular}

\section{TECHNIQUES GIVEN IN THE LITERATURE}

Two methods are given in the literature in order to construct discrete equispaced distributions.

Gerber and Jones (1976) propose a very intuitive rounding method. Suppose we want to transform the claim severity distribution $X$ into an equispaced distribution on $x=0, h, 2 h, \ldots$ The probabilities are simply given by

$$
\begin{aligned}
f_{X_{a p p}}(0) & =F_{X}\left(\frac{h}{2}-0\right) \\
f_{X_{a p p}}(x h) & =F_{X}\left(x h+\frac{h}{2}-0\right)-F_{X}\left(x h-\frac{h}{2}-0\right) \quad x=1,2, \ldots
\end{aligned}
$$

Suppose we choose $h=20$. We find the approached equispaced distribution $\left(X_{a p p}\right)$ :

TABLE 2

APPROACHED DISTRIBUTION; ROUNDING, $h=20$

\begin{tabular}{lcccc}
\hline$X_{a p p}$ & 0 & 20 & 40 & 60 \\
$f_{X_{\text {ajp }}}$ & 0.15 & 0.40 & 0.20 & 0.25 \\
\hline
\end{tabular}

The rounding method has the disadvantage that the approached distribution does not conserve any moment of the true distribution.

Gerber (1982) proposes a method that matches some moments: the local moment matching method (LMM).

Suppose for example we want to match two moments. Let the interval $\left(x_{k}, x_{k}+2 h\right]$. The masses $m_{0}^{k}, m_{1}^{k}, m_{2}^{k}$ are associated to the points $x_{k}, x_{k+h}, x_{k+2 h}$ in order to preserve the first two moments:

$$
\sum_{j=0}^{2}\left(x_{k}+j h\right)^{r} m_{j}^{k}=\int_{x_{k}}^{x_{k}+2 h} x^{r} d F_{X}(x) \quad r=0,1,2
$$


Using the Lagrange formula, Gerber (1982) gives the solution of the system (8):

$$
m_{j}^{k}=\int_{x_{k}}^{x_{k}+2 h} \prod_{i \neq j} \frac{x-x_{k}-i h}{(j-i) h} d F_{X}(x) \quad j=0,1,2
$$

In our case the integral is a sum.

The method might be applied in order to match more than two moments.

By taking $h=20$, we find

TABLE 3

INTERMEDIATE MASSES: LMM

\begin{tabular}{cccccc}
\hline \hline $\boldsymbol{m}_{\mathbf{0}}^{\mathbf{0}}$ & $\boldsymbol{m}_{\mathbf{1}}^{\mathbf{0}}$ & $\boldsymbol{m}_{\mathbf{2}}^{0}$ & $\boldsymbol{m}_{\mathbf{0}}^{\mathbf{1}}$ & $\boldsymbol{m}_{\mathbf{1}}^{\mathbf{1}}$ & $\boldsymbol{m}_{\mathbf{2}}^{\mathbf{1}}$ \\
\hline 0.1318 & 0.4389 & 0.0793 & 0.0836 & 0.2704 & -0.0040 \\
\hline \hline
\end{tabular}

and thus the following approached distribution:

TABLE 4

APPROACHED DISTRIBUTION: LMM. $h=20$

\begin{tabular}{llcccc}
\hline$X$ & 0 & 20 & 40 & 60 & 80 \\
$f_{X_{\text {app }}}$ & 0.1318 & 0.4389 & 0.1629 & 0.2704 & -0.0040 \\
\hline
\end{tabular}

This example shows an important disadvantage of the local moment matching method: the probabilities can take negative values.

With $h=17$, we find

TABLE 5

APPROACHED DISTRIBUTION; LMM, $h=17$

\begin{tabular}{llcccc}
\hline \hline$X$ & 0 & 17 & 34 & 51 & 68 \\
$f_{X_{\text {app }}}$ & 0.0998 & 0.4268 & 0.0921 & 0.3009 & 0.0804 \\
\hline
\end{tabular}

\section{The Kolmogorov distance}

The methods presented in the previous section are essentially local and they present some important disadvantages. In the sequel we will propose a global method consisting in the minimization of a distance between the true distribution and the approached distribution. 
Having chosen the equispaced points of the approached distribution, we minimize the distance in order to find the probabilities associated with the equispaced points.

The Kolmogorov distance between two random variables $X$ and $Y$ is given by

$$
d_{K}(X, Y)=\max _{x}\left|F_{X}(x)-F_{Y}(x)\right|
$$

where $F_{X}(x)$ denotes the cumulative distribution function of $X$.

This distance is used for the Kolmogorov-Smirnov adjustment test between two distributions. A small $d_{K}$ is in favour of the null hypothesis that the two distributions are the same while a large value of $d_{K}$ is in favour of the alternative hypothesis that the two distributions are different.

Suppose the same claim severity distribution as in the previous section. We want to approximate it by a distribution with masses at $0,20,40,60,80$ i.e. such that $h=20$. The problem is to find $f_{X_{a p p}(0)}, \ldots, f_{X_{a p p}}(80)$ in order to minimize $d_{K}\left(X, X_{a p p}\right)$. This is easily done numerically.

We find

TABLE 6

APPROACHED DISTRIBUTION; KOLMOGOROV, $h=20$

\begin{tabular}{llcccc}
\hline$X_{\text {app }}$ & 0 & 20 & 40 & 60 & 80 \\
$f_{X_{\text {app }}}$ & 0.2250 & 0.2976 & 0.2976 & 0.0899 & 0.0899 \\
\hline
\end{tabular}

$$
d_{K}\left(X, X_{a p p}\right)=0.175
$$

Other approached distributions might be obtained with the same Kolmogorov distance. This suggests that we might minimize $d_{K}$ under the constraint that the first moment of $X$ is preserved.

We then find

TABLE 7

APPROACHED DISTRIBUTION: KOLMOGOROV, $h=20$; FIRST MOMENT CONSTRAINED

\begin{tabular}{llcccc}
\hline \hline $\begin{array}{l}X_{\text {app }} \\
f_{X_{\text {cpp }}}\end{array}$ & 0 & 20 & 40 & 60 & 80 \\
\hline \hline & 0.225 & 0.2960 & 0.2604 & 0.1312 & 0.0874 \\
\hline \multicolumn{6}{c}{$d_{K}\left(X, X_{\text {app }}\right)=0.175$}
\end{tabular}


If the minimization is proceeded with the constraint of the first two moments, we find

TABLE 8

APPROACHED DISTRIBUTION; KOLMOGOROV, $h-21$; FIRST TWO MOMINTS CONSTRAINED

\begin{tabular}{cccccc}
\hline \hline$X_{\text {app }}$ & 0 & 20 & 40 & 60 & 80 \\
$f_{X_{c p p r}}$ & 0.1833 & 0.2500 & 0.4206 & 0.1156 & 0.0305 \\
\hline \hline
\end{tabular}

$$
d_{K}\left(X, X_{a p p}\right)=0.2167
$$

We see that in this case the Kolmogorov distance is higher with two moments constrained than with only one. The cost of matching several moments is such that the $d_{K}$ is higher. The preceding considerations suggest the following algorithm when using the Kolmogorov distance to construct an equispaced distribution:

1. Choose a set of equispaced points

2. $m=0$

3. Minimize $d_{K}\left(X, X_{a p p}\right)$ with $m$ moments constrained

4. Minimize $d_{K}\left(X, X_{a p p}\right)$ with $m+1$ moments constrained

5. If $d_{K}(m)=d_{K}(m+1)$ then $m=m+1$ and go to 4 else keep the approached distribution given by the minimization of $d_{K}(m)$.

6. Note that the necessity of keeping $n$ moments may overrule the rule 5

7. If the distance cannot be accepted then go back to 1 and change the equispaced points

Clearly the minimum may be achieved by more than one constrained distribution. This is not a problem in practice because the bounds we will derive for the distance between the compound distributions will depend only on the Kolmogorov distance between the distributions of $X$ and $X_{a p p}$.

\section{THE EFFECT OF THE APPROXIMATION ON THE COMPOUND DISTRIBUTION}

Let us now look at the Kolmogorov distance between the compound distribution $\left(f_{S}(x)\right)$ and the approached compound distribution $\left(f_{S_{u p p}}(x)\right)$. Suppose that $N$ is Poisson distributed $N \sim \operatorname{Po}(\lambda=0 . I)$.

The following distances have been calculated. 
TABLE 9

KOLMOGOROV DISTANCES WITH $\lambda=0.1$

\begin{tabular}{lcc}
\hline \hline & $\boldsymbol{d}_{\boldsymbol{K}}\left(\boldsymbol{X}, \boldsymbol{X}_{\text {app }}\right)$ & $\boldsymbol{d}_{\boldsymbol{K}}\left(\boldsymbol{S}, \boldsymbol{S}_{\text {app }}\right)$ \\
\hline$h=10 ; 4$ moments & 0.125 & 0.0115 \\
$h=10 ; 5$ moments & 0.125 & 0.0115 \\
$h=10 ; 6$ moments & 0.1273 & 0.0117 \\
$h=17 ;$ LMM & 0.1696 & 0.0157 \\
$h=17 ; 2$ moments & 0.1395 & 0.0131 \\
$h=20 ;$ Rounding & 0.25 & 0.0228 \\
$h=20 ; 1$ moment & 0.175 & 0.0161 \\
$h=20 ; 2$ moments & 0.2167 & 0.0202 \\
$h=20 ; 3$ moments & 0.2311 & 0.0212 \\
$h=25 ; 1$ moment & 0.225 & 0.0207 \\
$h=25 ; 2$ moments & 0.2646 & 0.0242 \\
\hline
\end{tabular}

We see that the $d_{K}\left(X, X_{u p p}\right)$ increases with $h$. This is not a surprise because, at the limit $h=0$, we would trivially have $d_{K}\left(X, X_{a p p}\right)=0$.

Concerning $d_{K}\left(S, S_{a p p}\right)$, the results are excellent. In each case, it is less than $10 \%$ of $d_{K}\left(X, X_{a p p}\right)$.

Of course the parameters of the claim frequency distribution must play a role. In our example, there is only one parameter: $\lambda$. Intuitively, we expect the $d_{K}\left(S, S_{a p p}\right)$ to grow with $\lambda$. The following calculations show it is not necessarily the case. find

We choose $h=10$ and 5 moments constrained $\left(d_{K}\left(X, X_{a p p}\right)=0.125\right)$. We

TABLE 10

KOLMOGOROV DISTANCES; $H=10$; FIRST FIVE MOMENTS CONSTRAINED

\begin{tabular}{ll}
\hline$\lambda$ & $d_{K}\left(\boldsymbol{S}, \boldsymbol{S}_{\text {app }}\right)$ \\
\hline 0.05 & 0.005992 \\
0.10 & 0.011488 \\
0.20 & 0.021113 \\
1 & 0.053471 \\
2 & 0.048496 \\
3 & 0.036778 \\
4 & 0.027632 \\
5 & 0.023072 \\
20 & 0.010845 \\
\hline
\end{tabular}


As this example shows, the $d_{K}\left(S, S_{a p p}\right)$ grows until around $\lambda=1$ and then decreases. In this example, the true distribution $X$ and the approached distribution $X_{a p p}$ are so closed that high order convolutions of the distributions remain very close. This explains why the Kolmogorov distance between the compound distributions decreases when $\lambda$ becomes very large.

Of course this is not the general rule. The following example shows that the Kolmogorov distance between the compound distributions increases with $\lambda$ when the approximation between $X$ and $X_{a p p}$ is bad:

TABLE 11

KOLMOGOROV DISTANCES; PATHOLOGICAL EXAMPLE,

\begin{tabular}{llll}
\hline \hline$X$ & 0 & 2 & 4 \\
$f_{X}$ & 0.4 & 0.2 & 0.4 \\
\hline
\end{tabular}

\begin{tabular}{lll}
\hline \hline$X_{\text {app }}$ & 0 & 3 \\
$f_{X_{\text {upp }}}$ & 0.3 & 0.7 \\
\hline
\end{tabular}

$$
d_{K}\left(X, X_{a p p}\right)=0.4
$$

\begin{tabular}{cc}
\hline \hline$\lambda$ & $\boldsymbol{d}_{\boldsymbol{K}}\left(\boldsymbol{S}, \boldsymbol{S}_{\text {app }}\right)$ \\
\hline 0.1 & 0.037062 \\
1 & 0.185621 \\
10 & 0.126143 \\
50 & 0.174345 \\
100 & 0.180262 \\
500 & 0.344425 \\
1000 & 0.464542 \\
\hline
\end{tabular}

5. UPPER AND LOWER BOUNDS FOR $d_{K}\left(S, S_{a p p}\right)$

We are going to use the following lemma (see De Pril and Dhaene (1992) for a proof):

$$
\text { If } d_{K}(X, Y) \leq a \text { then } d_{K}\left(X^{*^{n}}, Y^{*^{n}}\right) \leq n a
$$

With the analogous formula of (2) for the cdf's, we easily find an upper bound for the Kolmogorov distance between the true distribution and the approached distribution. 


$$
\begin{aligned}
d_{K}\left(S, S_{a p p}\right) & =\max _{x}\left|F_{S}(x)-F_{S_{a p p}}(x)\right| \\
& =\max _{x}\left|\sum_{n=0}^{\infty} p(n)\left(F_{X}^{*^{n}}(x)-F_{X_{a p p}}^{*^{n}}(x)\right)\right| \\
& \leq \sum_{n=0}^{\infty} p(n) \max _{x}\left|F_{X}^{*^{n}}(x)-F_{X_{a p p}}^{*^{n}}(x)\right| \\
& =\sum_{n=0}^{\infty} p(n) d_{K}\left(X^{*^{n}}, X_{a p p}^{*^{n}}\right) \\
& \leq \sum_{n=0}^{\infty} n p(n) d_{K}\left(X, X_{a p p}\right) \\
& =\mathbb{E} N d_{K}\left(X, X_{\text {app }}\right)
\end{aligned}
$$

A trivial lower bound is given by

$$
d_{K}\left(S, S_{a p p}\right) \geq\left|\Psi_{N}\left(\ln f_{X}(0)\right)-\Psi_{N}\left(\ln f_{X_{a p p}}(0)\right)\right|
$$

Obviously $d_{K}\left(X^{*^{n}}, X_{a p p}^{*^{n}}\right)$ is always $\leq 1$. Therefore the upper bound can be sharpened:

$$
\begin{gathered}
d_{K}\left(S, S_{a p p}\right) \leq \mathbb{E} N d_{K}\left(X, X_{a p p}\right) \\
-\sum_{n=2}^{\infty} p(n) \max \left(0, n d_{K}\left(X, X_{a p p}\right)-1\right)
\end{gathered}
$$

Let us take the example of table 10 :

TABLE 12

COMPARISON BETWEEN THE KOLMOGOROV DISTANCE AND THE BOUNDS; $h=10$

\begin{tabular}{ccccc}
\hline$\lambda$ & $\boldsymbol{d}_{\boldsymbol{K}}\left(\boldsymbol{S}, \boldsymbol{S}_{\text {app }}\right)$ & lower bound & upper bound & sharpened upper bound \\
\hline 0.1 & 0.011488 & 0.000712 & 0.0125 & 0.0125 \\
0.2 & 0.021113 & 0.001297 & 0.0250 & 0.025 \\
1 & 0.053471 & 0.003042 & 0.125 & 0.124999 \\
\hline
\end{tabular}

Even when $\lambda$ (or $\mathbb{E} N$ is a more general setting) is far from 0 , a precise bound can be obtained. There will be a cost for this operation: the calculation of some convolutions of $X$ and $X_{\text {app }}$.

Define $\Gamma_{\mathbb{E} N, a, b}=\{\lfloor\mathbb{E} N\rfloor-a ; \ldots ;\lfloor\mathbb{E} N\rfloor+b\}$ 
We have

$$
\begin{aligned}
d_{K}\left(S, S_{a p p}\right) \leq & \max _{x}\left|\sum_{j \in \Gamma_{\mathrm{E}, a, b}} p(j)\left(F_{X}^{*^{j}}(x)-F_{X_{a p p}}^{*^{\prime}}(x)\right)\right| \\
& +\sum_{j \in\left(\mathbb{Z}^{+} \backslash \Gamma_{\mathbb{E}, a, b}\right)} p(j) \min \left(1, j d_{K}\left(X, X_{a p p}\right)\right)
\end{aligned}
$$

TABLE 13

COMPARISON BETWEEN THE KOLMOGOROV DISTANCE AND THE BOUNDS; $h=10, \lambda=1$

\begin{tabular}{ll}
\hline$d_{K}\left(S, S_{a p p}\right)$ & 0.053471 \\
twice sharpened upper bound $\Gamma=\{1,2\}$ & 0.086197 \\
twice sharpened upper bound $\Gamma=\{1,3\}$ & 0.063503 \\
twice sharpened upper bound $\Gamma=\{1,4\}$ & 0.055846 \\
\hline
\end{tabular}

\section{THE DistancE OF TOTAL VARIATION}

Whenever you want to compare two integer random variables $X$ and $Y$, the total variation distance between the two variables is defined by

$$
d_{T V}(X, Y)=\sup _{A \subseteq Z^{+}}|P(X \in A)-P(Y \in A)|
$$

seems adequate. For example, this distance is very interesting when comparing the Poisson-Binomial and the Poisson distributions (see Barbour, Holst and Janson (1992)).

It is easy to show that the definition of the total variation distance is equivalent to

$$
d_{T V}(X, Y)=\frac{1}{2} \sum_{m=0}^{\infty}|P(X=m)-P(Y=m)|
$$

and so, it is immediate to deduce that

$$
d_{K} \leq d_{T V}
$$

In the present study, the total variation distance is uninteresting because it couldn't discriminate equispaced distributions. The reason for this fact is that only a few points share a positive probability for both the claim severity distribution and the approximate one.

This explains why we prefer to use the Kolmogorov distance than the total variation distance even if, at first side, it seems less general. 


\section{BERRY-ESSEEN BOUND FOR THE NORMAL APPROXIMATION}

In the preceding sections, we saw that whenever $\mathbb{E} N$ is high, the upper bound (15) is useless, even if, in practice, the approximation based on the Kolmogorov distance remains a good one. This fact, not really surprising, can be confirmed by a Berry-Esseen result.

Michel (1993) gives a Berry-Esseen bound for the normal approximation of a compound Poisson distribution. Then

Let $S$ a compound Poisson distribution. Assume the skewness of $S$ is finite.

$$
\sup _{t \in \mathbb{R}}\left|\mathbb{P}\left(\frac{S-\mathbb{E} S}{\sigma_{S}}\right)-\Phi(t)\right| \leq 0,8 \gamma_{S}
$$

where $\Phi(t)$ is the cumulative distribution of a standard normal distribution and

$$
\gamma_{S}=\frac{\mathbb{E} S^{3}-3 \mathbb{E} S \mathbb{E} S^{2}+2(\mathbb{E} S)^{3}}{\sigma_{S}^{3}} \text { is the skewness of } S
$$

For our numerical example, whenever $N$ is a Poisson random variable with mean $\lambda$, we obtain the following upper bound for the difference between the distribution of $S$ and the normal approximation:

TABLE 14

BERRY-ESSEEN BOUND IN FUNCTION OF $\lambda$

\begin{tabular}{rl}
$\lambda$ & bound \\
\hline 0,1 & 3,4965 \\
1 & 1,1057 \\
10 & 0,3496 \\
100 & 0,1105 \\
1000 & 0,0349 \\
\hline
\end{tabular}

The result is not surprising: whenever the mean of the counting distribution is low the approximation by the normal distribution is bad but in that case the method described in the previous section can be applied. Whenever this mean is high, the central limit approximation is good. Nevertheless, the techniques described in the previous sections remain interesting if one want an approximation of $S$ by discrete random variable $S_{a p p}$.

Assume the respective skewness are $\gamma$ and $\gamma_{a p p}$. If $X$ is a $N(\mathbb{E} S, \operatorname{Var} S)$ random variable, then by the Berry-Esseen result of Michel and the triangular inequality, we obtain

$$
\sup _{t \in \mathbb{R}}\left|\mathbb{P}(S<t)-\mathbb{P}\left(S_{a p p}<t\right)\right| \leq 0,8 \gamma+0,8 \gamma_{a p p}+\sup _{t \in \mathbb{R}}|\mathbb{P}[X<t]-\mathbb{P}[Y<t]|
$$


where $\quad X \sim N(\mathbb{E} S ; \mathbb{V a r} S)$

$$
Y \sim N\left(\mathbb{E} S_{a p p} ; \operatorname{Var} S_{a p p}\right)
$$

In particular, when $S_{a p p}$ is constructed in such a way that the first two moments of $X$ and $Y$ are the same, (18) becomes

$$
\sup _{t \in \mathbb{R}}\left|\mathbb{P}(S<t)-\mathbb{P}\left(S_{\text {app }}<t\right)\right| \leq 0,8 \gamma+0,8 \gamma_{\text {app }}
$$

Remarks:

1) Whenever the mean of $N$ is very large, a technical problem arises: the initialization of the recursion gives an underflow. This problem has been handled by Panjer and Wang (1995) but the technique they propose can not be controlled if one wants an arithmetic distribution. However if a normal approximation is sufficient, then it is very well controlled by Michel's (1993) result.

2) Michel (1993) gives also a non-uniform result under the same hypothesis:

$$
\left|\mathbb{P}\left(\frac{S-\mathbb{E} S}{\sigma_{S}}\right)-\Phi(t)\right| \leq \frac{30,6}{1+|t|^{3}} \gamma_{S}
$$

Unluckily this result is more interesting than the uniform bound only if $|t| \geq 3,34$, i.e. in the very far tail of the distribution. This is the reason why we concentrate only on the uniform result. Of course the nonuniform result of $\mathrm{Michel}$ is interesting to use when approximating quantities like stop-loss premiums for example.

\section{EXTENSION}

The proof of Michel's (1993) result is given for the case where $N$, the number of components of the random sum, is a Poisson random variable but as the proof shows, the crucial point for the extension of the Berry-Esseen result to the random case is the fact that the distribution of $S$ is infinitely divisible. Consequently, $N$ has to be infinitely divisible.

In fact, by Feller (1971), there is a logical equivalence between infinite divisibility on integers and Compound Poisson distributions.

Then the result of Michel (1993) is immediately extended to every infinite divisible distribution on integers.

As an example of more general distribution for $N$, we take the Hofmann's family of distributions (see Hofmann (1955) and Kestemont and Paris (1985) for a discussion) whose probability generating function is

$$
\Psi_{N}(z)=e^{-\frac{p}{c(1-a)}\left((1+c(1-z))^{1-a}-1\right)}
$$

This distribution depends on three parameters: $p, c$ and $a$. The parameter $a$ distinguishes the distributions and presents the particular cases: Poisson 
$(a=0)$, Poisson Inverse Gaussian $(a=1 / 2)$, Binomial Negative $(a=1)$, Polya-Aeppli $(a=2)$ and Neymann Type A $(a \rightarrow \infty, c \rightarrow \infty, a c \rightarrow b)$.

The mean of the distribution is $p$ and has for maximum likelihood estimator the experimental mean.

This distribution is also known as the Generalized Poisson Pascal distribution (see Panjer and Willmot (1992) but we prefer the Hofmann's presentation which has a better parametrization and natural extensions (see Walhin and Paris (1998)).

The Hofmann distribution $\operatorname{Ho}(p, c, a)$ is itself a compound Poisson distribution and you can write

$$
N=\xi_{1}+\ldots+\xi_{L}
$$

where $L$ is poisson distributed

$$
\xi \text { is such that } \frac{\mathbb{P}[\xi=n]}{\mathbb{P}[\xi=n-1]}=a+\frac{b}{n} \quad n \geq 2
$$

Then the generalization of Panjer's recursion (Sundt and Jewell (1981)) can be used when $\xi$ is a counting distribution. The aggregate claims distribution can then be evaluated by a double application of Panjer's recursion because

$$
\begin{aligned}
& V=X_{1}+\ldots+X_{\xi} \\
& S=V_{1}+\ldots+V_{L}
\end{aligned}
$$

(see Panjer and Willmot (1992) for a general statement and Walhin and Paris (1998) for details concerning the Hofmann's distribution).

As $N$ is infinitely divisible (see (19)), the results described in the previous section will apply for this general counting distribution.

The characteristics of the distribution are

$$
\begin{aligned}
S & =X_{1}+\ldots+X_{N} \\
N & \sim H o(p, c, a) \\
\mu_{1} & =\mathbb{E} X \\
\mu_{2} & =\mathbb{E} X^{2} \\
\mu_{3} & =\mathbb{E} X^{3} \\
\operatorname{Var} S & =\left(c a \mu_{1}^{2}+\mu_{2}\right) p \\
\gamma_{S} & =\frac{a c^{2} \mu_{1}^{3}+a^{2} c^{2} \mu_{1}^{3}+3 a c \mu_{1} \mu_{2}+\mu_{3}}{\left(a c \mu_{1}^{2}+\mu_{2}\right)^{\frac{3}{2}}} p^{-\frac{1}{2}}
\end{aligned}
$$




\section{CONCLUSION}

We have seen how a global method can give an equispaced approached distribution of a general discrete distribution. The minimization of the Kolmogorov distance does not present the disadvantages of the methods presented in the literature. The problem of matching moments between both distributions can be solved.

When the mean of the frequency claim distribution is low, which is typically the case in automobile insurance, the knowledge of the Kolmogorov distance between both the severity distributions is sufficient to give a sharp upper bound on the Kolmogorov distance between both the compound distributions.

When the mean of the counting distributions is high, there exists an interesting Berry-Esseen bound for the normal approximation when $N$ is infinitely divisible. If one wants to keep the discrete approximate distribution, the Berry-Esseen bound may be used twice in order to give a bound on the Kolmogorov distance between the true and approximate compound distributions.

When the mean of the claim frequency is not high enough for using the Berry-Esseen result, the bounds can be sharpened at the price of calculating some high order convolutions of both the true and approximate severity distributions. Then almost every situation can be handled, in term of controlling the error induced, by the techniques described in this paper. Note that the two practical situations, events with very low frequency and very large portfolios are very well controlled.

Our experience shows that even when the mean of the frequency claim distribution is medium, the Kolmogorov distance between both compound distributions remains a fraction of the Kolmogorov distance between both the claim severity distributions if the approximation is good. Note that the properties of the Kolmogorov distance between the compound distributions can also be used in the case of a bad knowledge of the claim severity distribution.

\section{ACKNOWLEDGMENT}

We thank an anonymous referee for pointing out the interest of BerryEsseen type results.

\section{REFERENCES}

Barbour, A.D., Holst, L., and Janson, S. (1992). Poisson Approximation. Oxford University Press.

DE PRIL, N. (1985). Recursions for Convolutions of Arithmetic Distributions. ASTIN Bulletin. 15: $135-139$.

De Pril, N. and Dhaene, J. (1992). Error Bounds for Compound Poisson Approximations of the Individual Risk Model. ASTIN Bulletin, 22: 137-148.

Feller, W. (1971). An Introduction to Probability Theory and its Applications Vol II (3ed). Wiley, New York. 
Gerber, H.U. (1982). On the Numerical Evaluation of the Distribution of Aggregate Claims and its Stop-Loss Premiums. Insurance: Mathematics and Economics, 1: 13-18.

Kestemont, R.M. and Paris, J. (1985). Sur l'Ajustement du Nombre de Sinistres. Bulletin of the Swiss Actuaries, 85: 157-163.

Michel, R. (1993). On Berry-Esseen Results for the Compound Poisson Distribution. Insurance: Mathematics and Economics, 13: 35-37.

PANJER, H.H. (1981). Recursive Evaluation of a Family of Compound Distributions. ASTIN Bulletin, 12: 22-26.

PANJer, H.H. and Lutrk, B.W. (1983). Practical Aspects of Stop-Loss Calculations. Insurance: Mathematics and Economics, 2: 159-177.

PANJER, H.H. and Willmot, G.E. (1992). Insurance Risk Models. Society of Actuaries.

VAN BeEk, P. (1972). An Application of Fourier Methods to the Problem of Sharpening the Berry-Esseen Inequality. Z. Wahrscheinlichkeitstheorie verw. Geb., 23: 187-196.

Walhin, J.F. and Paris, J. (1998). A Large Family of Discrete Probability Laws. forthcoming.

WANG, S. and PANJer, H.H. (1994). Proportional Convergence and Tail-Cutting Techniques in Evaluating Aggregate Claim Distributions. Insurance: Mathematics and Economics, 14: 129-138.

\section{JEAN-FranÇOIS WALHIN**}

José Paris*

* Institut de Statistique

Voie du Roman Pays, 20

B-1348 Louvain-la-Neuve

BELGIQUE

- Le Mans Assurances

A venue Louise, 222

B-1050 Bruxelles

BELGIQUE 